\title{
Investigation of Human IFITM3 Polymorphisms rs34481144A and rs12252C and Risk for Influenza A(H1N1)pdm09 Severity in a Brazilian Cohort
}

\section{OPEN ACCESS}

Edited by:

Georges Michel Verjans, Erasmus Medical Center, Netherlands

Reviewed by:

Linda M. Wakim,

The University of Melbourne, Australia

Chen Liang,

McGill University, Canada

*Correspondence:

Aline R. Matos

aline.matos@ioc.fiocruz.br

Specialty section:

This article was submitted to

Virus and Host,

a section of the journal

Frontiers in Cellular and Infection

Microbiology

Received: 02 March 2020

Accepted: 08 June 2020

Published: 10 July 2020

Citation:

Martins JSC, Oliveira MLA, Garcia CC,

Siqueira MM and Matos AR (2020)

Investigation of Human IFITM3

Polymorphisms rs34481144A and rs12252C and Risk for Influenza $A($ H1N1)pdm09 Severity in a Brazilian

Cohort.

Front. Cell. Infect. Microbiol. 10:352. doi: 10.3389/fcimb.2020.00352

\author{
Jéssica S. C. Martins ${ }^{1}$, Maria L. A. Oliveira ${ }^{2}$, Cristiana C. Garcia ${ }^{1}$, Marilda M. Siqueira ${ }^{1}$ \\ and Aline R. Matos ${ }^{1 *}$
}

'Laboratório de Virus Respiratórios e do Sarampo, Instituto Oswaldo Cruz (Fiocruz), Rio de Janeiro, Brazil, ${ }^{2}$ Laboratório de Desenvolvimento Tecnológico em Virologia, Instituto Oswaldo Cruz (Fiocruz), Rio de Janeiro, Brazil

Influenza is a major public health problem that causes acute respiratory infection in humans. Identification of host factors influencing in disease outcome is critical for recognition of individuals with increased risk. Investigations on the role of rs34481144A and rs12252C IFITM3 polymorphisms in influenza $\mathrm{A}(\mathrm{H} 1 \mathrm{N1})$ pdm09 severity is not yet conclusively determined. This study aimed to evaluate such polymorphisms frequencies and IFITM3 levels in an infected Brazilian cohort of 314 influenza $A(\mathrm{H} 1 \mathrm{~N} 1)$ pdm09 cases and its putative association with clinical, epidemiological and virological data. Individuals were clinically classified into mild, severe and fatal cases. IFITM3 polymorphisms were detected by specific Taqman probes in real time PCR reactions. IFITM3 levels were determined by quantitative real time PCR. Thus, the different clinical groups presented similar distribution of rs34481144 and rs12252 genotypes and allelic frequencies. There was no significant association between the polymorphisms with severity of disease by using distinct genetic models. Additionally, geographic distribution of mutants showed that rs34481144A allele was more predominant in Brazilian Southern region. In contrast, rs12252C allele presented similar frequencies in all regions. Individuals with the distinct rs34481144 and rs12252 genotypes showed similar levels of IFITM3 and viral load in their respiratory specimens. Furthermore, IFITM3 levels were comparable in the distinct clinical groups and were not correlated with influenza viral load in analyzed samples. Thereby, rs34481144A and rs12252C polymorphisms were not associated with severity or mortality of influenza $A(\mathrm{H} 1 \mathrm{~N} 1)$ pdm09 infection nor with IFITM3 transcript levels and influenza viral load in upper respiratory tract samples in a Brazilian cohort.

Keywords: influenza, biomarker, IFITM3, polymorphism, susceptibility

\section{INTRODUCTION}

Influenza viruses cause acute respiratory infections in humans, which are correlated to annual epidemics and occasional pandemics, representing a relevant public health problem (Taubenberger and Morens, 2008). Annually, an estimated one billion cases of influenza, of which 3-5 million are severe cases, result in 300,000-650,000 deaths, worldwide (Danielle Iuliano et al., 2018; World Health Organization, 2019). Children under 5 years old, the elderly, individuals with chronic 
diseases and immunocompromised are more prone to develop severe disease, composing the standard risk groups for this infection (Cox and Subbarao, 1999). Influenza A(H1N1)pdm09 and $\mathrm{H} 3 \mathrm{~N} 2$ subtypes, as well as B viruses, are the current major sources of human influenza infections. Currently, vaccination and antiviral treatment are the main control measures of this infection. However, due to the viral evolutionary patterns, vaccines must be updated regularly (Epperson et al., 2019). In addition, identification of influenza strains that are resistant to the current antiviral drugs has been reported, yet still in low levels (Matos et al., 2018; Takashita et al., 2020).

Because influenza A virus infect distinct animal hosts, genetic shift can eventually occur, arising genetic and antigenically distinct reassortant viruses. Depending on their ability of inter-individual transmission within the human susceptible population, these emerging viruses pose a potential pandemic risk, which occurred in 2009, with the introduction of influenza $\mathrm{A}(\mathrm{H} 1 \mathrm{~N} 1)$ pdm09 virus into the human population. During this period, healthy young adults were severely affected and counted for a significant portion of the fatalities (Taubenberger and Morens, 2008).

The clinical evolution of influenza infection can be influenced by multiple factors, including individual susceptibility, pathogen virulence and host pre-existing antibody titers. Influenza virulence can be altered by acquisition of mutations during virus evolutionary process that can modify its interactions with the infected cell, increasing viral affinity for cell receptors (Chutinimitkul et al., 2010; Liu et al., 2010), and by influencing host immune response (Fislová and Kostolanský, 2005). Likewise, viruses can evade immune response conferred by antibodies produced during previous infections and by vaccination (van der Sandt et al., 2012). Similarly, host genetic variants may also affect modulation of immune response after viral infection (Falcon et al., 2015; Chan et al., 2017). Putative associations between genetic polymorphisms in host crucial genes that act against virus infection that are essential for virus-host interplay have been recently explored regarding their association with susceptibility/severity of influenza infections (Zhou et al., 2012; Tarnow et al., 2014; Cheng et al., 2015; Chan et al., 2017; Garcia et al., 2018; Matos et al., 2019).

Interferon-induced transmembrane protein three (IFITM3) is a proinflammatory cytokine belonging to the group of interferon (IFN) stimulated genes (ISGs), which are induced after viral infection. The process of induction of ISGs starts following the fusion of viral envelope with the cell endosomal membrane, when the viral genetic material is released into the cytoplasm and allows its recognition by cellular pattern recognition receptors (PRRs) (Heil et al., 2004; Kawai and Akira, 2010; Kim et al., 2016) that stimulate secretion of type I IFNs, which, in turn, bind to their receptor (IFNAR) and activate the JAK/STAT pathway, driving ISGs expression. IFITM3 presents an influenza restriction function by preventing formation of fusion pores necessary for the release of viral genetic material into the cytoplasm, thus abrogating virus replication (Feeley et al., 2011; Huang et al., 2011; Suddala et al., 2019). Complementarily, it has been described that ifitm3 knockout mice show higher levels of influenza replication and develop fulminant viral pneumonia (Everitt et al., 2013).

Because of its central role in influenza restriction (Brass et al., 2009; Feeley et al., 2011; Everitt et al., 2013; Desai et al., 2014), single nucleotide polymorphisms (SNPs) in IFITM3 gene have been associated with an increased severity of influenza A(H1N1)pdm09 infection (Randolph et al., 2017; Allen et al., 2018; Zani and Yount, 2018; Kim et al., 2019). One of the investigated SNPs, the rs34481144A, leads to a substitution in IFITM3 promoter region (Randolph et al., 2017) and a further modification of a methylation site. These events increase the affinity for the CTCF transcriptional factor, which could interfere with IFITM3 transcription. The presence of this mutation was previously associated with lower IFITM3 expression levels and decreased binding affinity for the regulatory factor IRF3. A previous relationship of this SNP with higher risk of severe influenza infection has been described (Allen et al., 2018). In addition, the rs $12252 \mathrm{C}$ is described as responsible for generating a truncated protein, lacking the initial 21 amino acids of the $\mathrm{N}$-terminal region ( $\Delta 21$ IFITM3) (David et al., 2018). The deleted region comprises the regulatory YEML internalization motif recognized by the AP-2 complex, that conducts IFITM3 localization into late endosomes, multivesicular bodies and lysosomes (Chesarino et al., 2014; Jia et al., 2014). Moreover, the PPNY motif, also included in the deleted region, recruits NEDD4 to promote IFITM3 ubiquitination and turnover via lysosomes (Chesarino et al., 2015). Therefore, rs12252C modifies IFITM3 intracellular localization and levels which, consequently, interfere with restriction against influenza virus (Everitt et al., 2013; Compton et al., 2016). Despite that, some studies have shown that patients that present rs12252C in homozygosity displayed the majority of IFITM3 transcripts as the complete isoform (Randolph et al., 2017; Makvandi-Nejad et al., 2018). However, the association of these polymorphisms and the risk of severe influenza is not totally clear (Zhang et al., 2013; Kim and Jeong, 2017; Pan et al., 2017; Randolph et al., 2017; David et al., 2018).

In this study, we report the distribution of IFITM3 rs34481144A and rs12252C polymorphisms in a Brazilian cohort of influenza $\mathrm{A}(\mathrm{H} 1 \mathrm{~N} 1) \mathrm{pdm} 09$ positive cases, whose samples were collected during the 2012-2018 period, further classified according to their clinical presentation as mild, severe and fatal infections. Furthermore, we explored putative relationships between these SNPs with clinical, epidemiological and virological variables, in addition to IFITM3 expression levels.

\section{MATERIALS AND METHODS}

\section{Population}

Our laboratory is a National Reference Laboratory part of the Influenza Surveillance System (ISS) for the Brazilian Ministry of Health and is one of the National Influenza Centers (NICs) of the World Health Organization (WHO) (WHO, 2020). Thus, we systematically receive samples from nine out of 27 Brazilian states (Southern, Northeastern and Southern regions). During the 2012-2018 period, approximately 25,000 influenza A(H1N1)pdm09 cases were investigated by the Brazilian ISS. 
Almost $10 \%$ of these samples were received at our NIC (Fiocruz, Rio de Janeiro). In this study, we included 314 respiratory clinical samples that were collected from each patient as three individual swabs from the two nostrils and the oropharynx. In this type of samples, we mostly detect epithelial cells (Daley et al., 2006). Inclusion criteria consisted of the availability of clinical and epidemiological records, which were collected by ISS teams from each Brazilian State. Further, cases were classified into influenza-like illness (ILI), severe acute respiratory infection (SARI) and fatal cases. ILI was defined as presence of fever (even if reported) and cough or sore throat, plus one of the following symptoms: headache, myalgia or arthralgia. SARI cases were defined as cases requiring hospitalization and presenting dyspnea or one of the following signs of severity: peripheral capillary oxygen saturation $<95 \%$, respiratory distress or acute respiratory insufficiency (Secretaria de Vigilância em Saúde, 2012). This study was approved by Fiocruz-IOC Ethics Committee, approval number 2.453.470.

\section{Influenza Detection}

Total RNA was extracted from clinical respiratory samples by using the QIAmp Viral RNA Mini kit (Qiagen, Germany), according to the manufacturer's instructions. For influenza A(H1N1)pdm09 detection, extracted RNA was tested in a onestep real time RT-PCR assay using specific primers and probes (CDC, USA), as recommended by the WHO. Influenza viral load was indirectly determined by real-time RT-PCR at the moment of diagnosis as the CT (cycle threshold) number during its detection (Behillil et al., 2018; Mares-Guia et al., 2020).

\section{Genotyping}

Genomic DNA was extracted from clinical respiratory samples by using the PureLink ${ }^{\mathrm{TM}}$ Genomic DNA Mini Kit (Invitrogen, USA), as previously described (Garcia et al., 2018; Matos et al., 2019). After that, the rs $34481144 \mathrm{~A}$ and rs12252C polymorphisms were identified using pre-defined TaqMan ${ }^{\mathrm{TM}}$ SNP Genotyping Assays probes (assays IDs: C__26288451_10 and C_175677529_10) (Thermo Fisher Scientific, USA) in a real time PCR assay performed in the Step One Plus equipment (Applied Biosystems, USA) with 5-20 ng DNA, per sample, according to manufacturer's instructions. Cycling parameters were as follows: $95^{\circ} \mathrm{C}$ for $10 \mathrm{~min}$, followed by 50 cycles of $95^{\circ} \mathrm{C}$ for $15 \mathrm{~s}$ and $60^{\circ} \mathrm{C}$ for $90 \mathrm{~s}$. In this methodology, each TaqMan probe presents a distinct fluorescent signal and is specific for amplification of a different nucleotide at each position. In the end of the run, the software groups the samples according to their specific unique (homozygotes) or mixed (heterozygotes) fluorescence signal, as previously described (Randolph et al., 2017; Garcia et al., 2018).

\section{Quantification of IFITM3 Expression}

After total RNA extraction, we proceeded to reverse transcription with oligo (dT) primers and Superscript III Reverse Transcriptase (Thermo Fisher Scientific, USA). Quantitative real time PCR was carried out in a real time PCR Step One Plus equipment (Applied Biosystems, USA) with the following specific primer sequences for IFITM3: F-5' GGTCTTCGCTGGACACCAT 3' and $\mathrm{R}-5^{\prime}$ TGTCCCTAGACTTCACGGAGTA $3^{\prime}$. All reactions were conducted using the SYBR Green detection reagent (Applied Biosystems, USA) and were carried out in duplicates. Cycling conditions consisted of the initial steps of $50^{\circ} \mathrm{C}$ for $2 \mathrm{~min}$ and $94^{\circ} \mathrm{C}$ for $5 \mathrm{~min}$, followed by 40 cycles of $94^{\circ} \mathrm{C}$ for $30 \mathrm{~s}, 50^{\circ} \mathrm{C}$ for $30 \mathrm{~s}$ and $72^{\circ} \mathrm{C}$ for $45 \mathrm{~s}$. A final melting curve analysis was performed to determine amplification specificity $\left(60-95^{\circ} \mathrm{C}\right.$ with a heating rate of $0.2^{\circ} \mathrm{C} / \mathrm{s}$ and continuous fluorescence measurement). Expression data were normalized to glyceraldehyde 3-phosphate dehydrogenase (GAPDH) expression and analyzed by using the $2^{-\Delta \Delta C T}$ method.

\section{Statistical Analysis}

The Hardy-Weinberg equilibrium (HWE) for the allele frequency and genotype distribution was assessed by using the web program (http://www.oege.org/software/hwe-mr-calc.shtml) (Rodriguez et al., 2009), and deviation from HWE was assessed by Chi-square test. After descriptive analyses, putative associations between outcomes (genotypes) and clinical/epidemiological traits were explored (Chi-square or Fisher's exact test for discrete variables and ANOVA for means) using SPSS software for Windows, version 19.0 (IBM Inc., USA). Additional statistical analysis was performed with GraphPad Prism (GraphPad Software Inc., USA). IFITM3 expression level and influenza viral load values, in the clinical or genotyped groups, were compared by Student's $T$ test or One-Way ANOVA with Bonferroni post-test for multiple comparisons. Finally, correlation between IFITM3 expression and influenza viral load were assessed by linear regression to calculate $\mathrm{R}^{2}$ and $p$-values. Results were considered significant when $p<0.05$.

\section{RESULTS}

\section{Clinical, Demographical and Epidemiological Characteristics of the Enrolled Brazilian Patients}

In this study, we investigated 314 patients positive for influenza A(H1N1)pdm09 infection during the 2012-2018 period. Individuals were classified according to their infection severity as ILI $(n=92)$, SARI $(n=140)$ or fatal $(n$ $=82$ ) cases, as mentioned in the methodology section. Of note, influenza $\mathrm{A}(\mathrm{H} 1 \mathrm{~N} 1) \mathrm{pdm} 09$ virus was the most prevalent subtype among the Brazilian cases from this period (data from Brazilian ISS, available at http://portalms.saude.gov.br/ saude-de-a-z/gripe/situacao-epidemiologica-dados). Therefore, $51.6 \%$ of the patients were male and the median age of the individuals was 39 years old (Table 1). Moreover, the median age of the patients in the ILI, SARI and fatal groups were significantly different $(29,43$, and 46 years old, respectively; $p<0.01$ ), as already expected, since the higher the age, the greater the risk of disease complications (Cox and Subbarao, 1999). Among SARI patients, $88.1 \%$ presented dyspnea, $71.3 \%$ respiratory distress and $46.1 \%$ oxygen saturation $<95 \%$. Also, $53.1 \%$ of the patients informed any comorbidity (pneumopathy, cardiopathy, immunodepression, hypertension, hepatopathy, neurologic disorders, nephropathy, diabetes, cancer, obesity and 
TABLE 1 | Clinical, demographical and epidemiological characteristics of the influenza A(H1N1)pdm09 Brazilian patients whose samples were included in this study.

\begin{tabular}{|c|c|c|c|c|c|}
\hline Characteristics & Total & ILI & SARI & Fatal & $p$-value \\
\hline Number of samples & 314 & 92 & 140 & 82 & NA \\
\hline Age (years) - median \pm SD & $39 \pm 22.6$ & $29 \pm 18.5$ & $43 \pm 24.8$ & $46 \pm 20.7$ & $<0.01$ \\
\hline Male-n (\%) & $162(51.6)$ & $52(56.5)$ & $63(45.0)$ & $47(57.3)$ & 0.11 \\
\hline \multicolumn{6}{|l|}{ Signs and symptoms } \\
\hline Cough-n/N (\%) & 246/255 (96.5) & 66/69 (95.6) & 138/140 (98.6) & 42/46 (91.3) & 0.06 \\
\hline Sore throat－n/N (\%) & 90/199 (45.2) & 38/64 (59.4) & 43/105 (41.0) & 9/30 (30.0) & 0.01 \\
\hline Coryza-n/N (\%) & 21/161 (13.0) & $14 / 58(24.1)$ & $5 / 76(6.6)$ & $2 / 27(7.4)$ & $<0.01$ \\
\hline Dyspnea-n/N (\%) & $165 / 184(89.7)$ & NA & 119/135 (88.1) & 46/49 (93.9) & 0.25 \\
\hline Respiratory distress - n/N (\%) & 111/160 (69.4) & NA & $82 / 115(71.3)$ & $29 / 45(64.4)$ & 0.39 \\
\hline Oxygen saturation < 95\% - n/N (\%) & $77 / 160(48.1)$ & NA & $53 / 115(46.1)$ & $24 / 45(53.3)$ & 0.40 \\
\hline Comorbidities - n/N (\%) & 95/179 (53.1) & 9/37 (24.3) & 65/109 (59.6) & 21/33 (63.6) & $<0.01$ \\
\hline \multicolumn{6}{|l|}{ Geographic region } \\
\hline South -n (\%) & 166 (52.9) & $67(72.8)$ & $49(35.0)$ & $50(61.0)$ & $<0.01$ \\
\hline Southeast-n (\%) & $97(30.9)$ & $9(9.8)$ & $67(47.8)$ & $21(25.6)$ & \\
\hline Northeast-n (\%) & $51(16.2)$ & $16(17.4)$ & $24(17.1)$ & $11(13.4)$ & \\
\hline
\end{tabular}

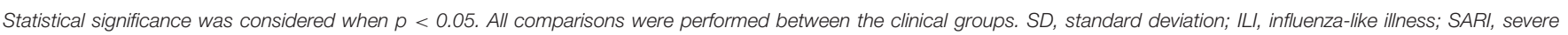
acute respiratory infection; NA, not applicable; $n$, number of cases with the referred characteristic; $N$, number of cases for which the information is available.

smoking), and they were less predominant in the ILI group, as compared to SARI and fatal groups $(24.3,59.6$, and $63.6 \%$, respectively; $p<0.01$ ), considering that comorbidities are also risk factors for complications. Regarding demographical origin of the samples, most of them were collected at the Brazilian Southern region, followed by the Southeastern and the Northeastern regions (52.9, 30.9, and 16.2\%, respectively; $p<0.01$; Table 1).

\section{Relationship of IFITM3 Polymorphisms rs34481144A and rs12252C and Severe Influenza A(H1N1)pdm09}

In our Brazilian cohort, the frequencies of IFITM3 rs34481144A and rs $12252 \mathrm{C}$ genotypes were in accordance with $\mathrm{HWE}(p>$ 0.05). The overall IFITM3 rs34481144A allelic frequency was $31.0 \%$ (Figure 1A). The distribution of rs3448144 genotypes was as follows: $47.8 \%$ of the individuals had the GG wild type (WT), whereas $42.4 \%$ had the GA heterozygous genotype and 9.9\% had the AA mutant homozygous genotype (Figure 1B). There was no difference in the distribution of rs34481144 genotypes and alleles between the ILI, SARI and fatal groups, when using additive, dominant, recessive and allelic genetic models $(p=0.92, p=0.88, p=0.71$, and $p=0.83$, respectively; Table 2). Distinct stratification of the clinical groups (ILI vs. SARI + fatal or ILI + SARI vs. fatal) also failed to identify association of rs34481144A with severity or mortality.

Concerning the rs $12252 \mathrm{C}$ polymorphism, we detected a total allelic frequency of $16.4 \%$ (Figure 1C). Rs12252 genotype distribution was $71.0 \%$ for WT (TT), 25.2\% for heterozygous (CT) and $3.8 \%$ for mutant homozygous (CC) (Figure 1D). In a similar fashion to rs34481144, we did not observe differences in rs12252 genotypes and allelic distributions between the distinct clinical groups of influenza cases by assuming the distinct genetic models (Table 2). Noteworthy, the rare CC genotype was not identified in any of the fatal cases.

In our analysis, the rs34481144 and rs12252 alleles were not associated with age, gender, clinical symptoms and comorbidities, suggesting that their frequencies are not influenced by these factors.

Intriguingly, the presence of rs34481144-AA and rs12252-CC homozygous mutant genotypes concomitantly was not detected in any of the influenza cases in our cohort $(p<0.001)$. The rs3448114-AA recessive genotype was always transmitted with the rs12252-TT dominant genotype, and the rs12252-CC recessive genotype was ever inherited with the rs34481144-GG dominant genotype in our influenza cases. By contrast, both dominant genotypes (GG and TT) could be inherited with any of the other genotypes. These results suggest that their presence at homozygosity does occur simultaneously or consists of a rare event.

\section{Geographical Distribution of rs12252C and rs34481144A Polymorphisms}

In Brazil, distinct geographical regions present dissimilar patterns of genetic ancestry due to specific migratory currents from distinct continents (Alves-Silva et al., 2000; Mychaleckyj et al., 2017). In the light of this knowledge, we investigated SNPs allelic and genotypes distribution in the influenza cases according to their geographical Brazilian region. Therefore, we observed that rs34481144A allelic prevalence was higher in the Brazilian Southern region, followed by the Southeastern and Northeastern regions (34.6; 29.4, and $22.5 \%$, respectively; $p=0.05)$. In contrast, rs12252C allele presented similar patterns of predominance in the Southern, Southeastern and Northeastern regions (14.1, 19.1, and 37.7\%, respectively; $p=0.29$ ). 


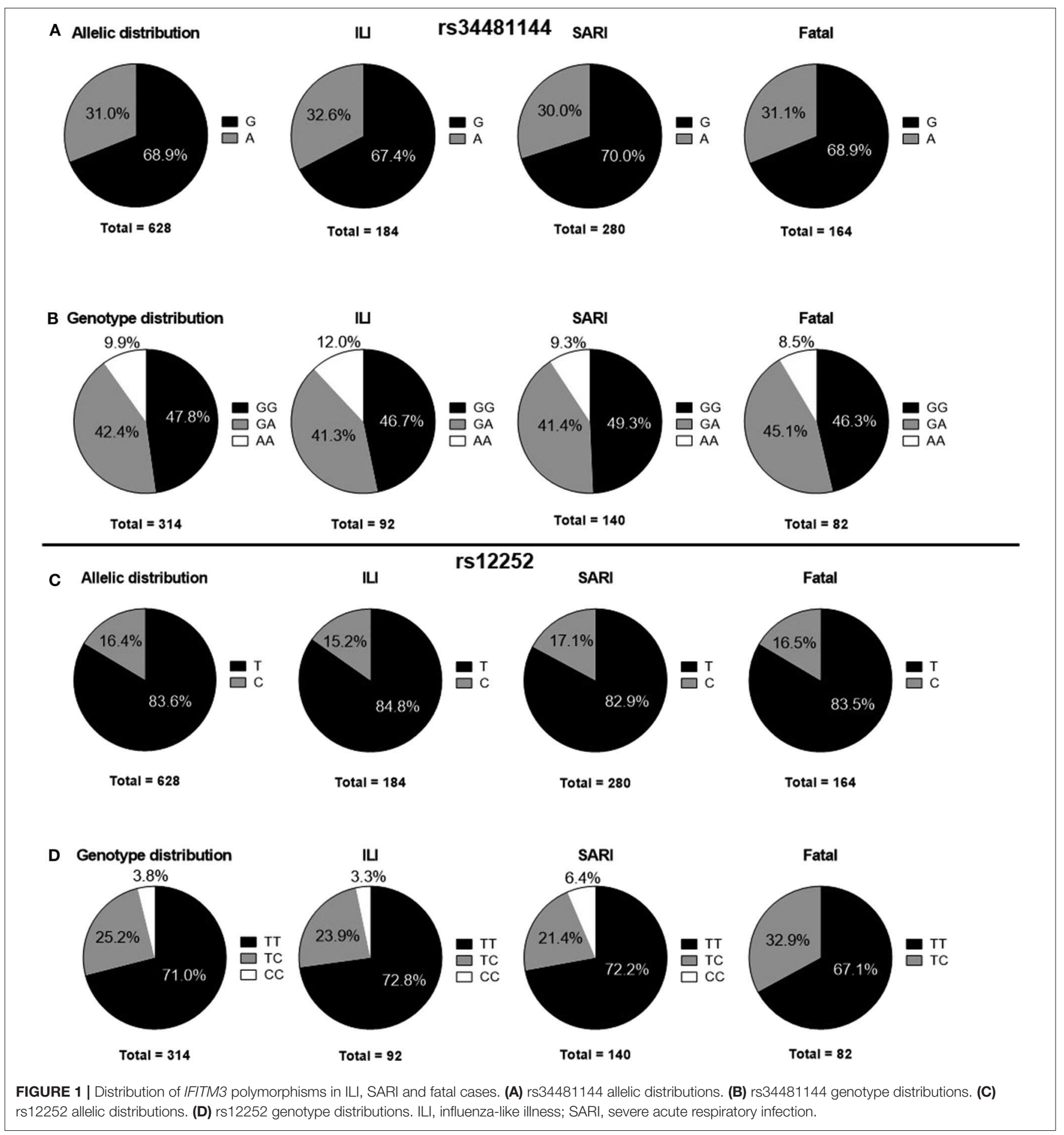

\section{Analysis of Association of Polymorphisms With IFITM3 Expression and Influenza Viral Load}

Because it was previously shown that rs34481144A and rs12252C could interfere with IFITM3 expression, we analyzed its transcript levels in our genotyped influenza positive clinical specimens. As a result, IFITM3 presented comparable levels among distinctly genotyped rs34481144 and rs12252 subjects
( $p=0.69$ and 0.20 , respectively; Figures $2 \mathbf{A}, \mathbf{B})$. Of note, as rs12252-CC genotype was detected in a low frequency in our cohort (3.8\%, Figure 1D), material was not enough to perform robust expression analysis.

Additionally, we further explored a possible correlation between rs34481144 and rs12252 genotypes and influenza viral load, but we did not detect any significant difference in our positive cases (Figures 2C,D). Furthermore, as IFITM3 
TABLE 2 | Distinct models for the risk assessment of IFITM3 rs34481144 and rs12252 genotypes.

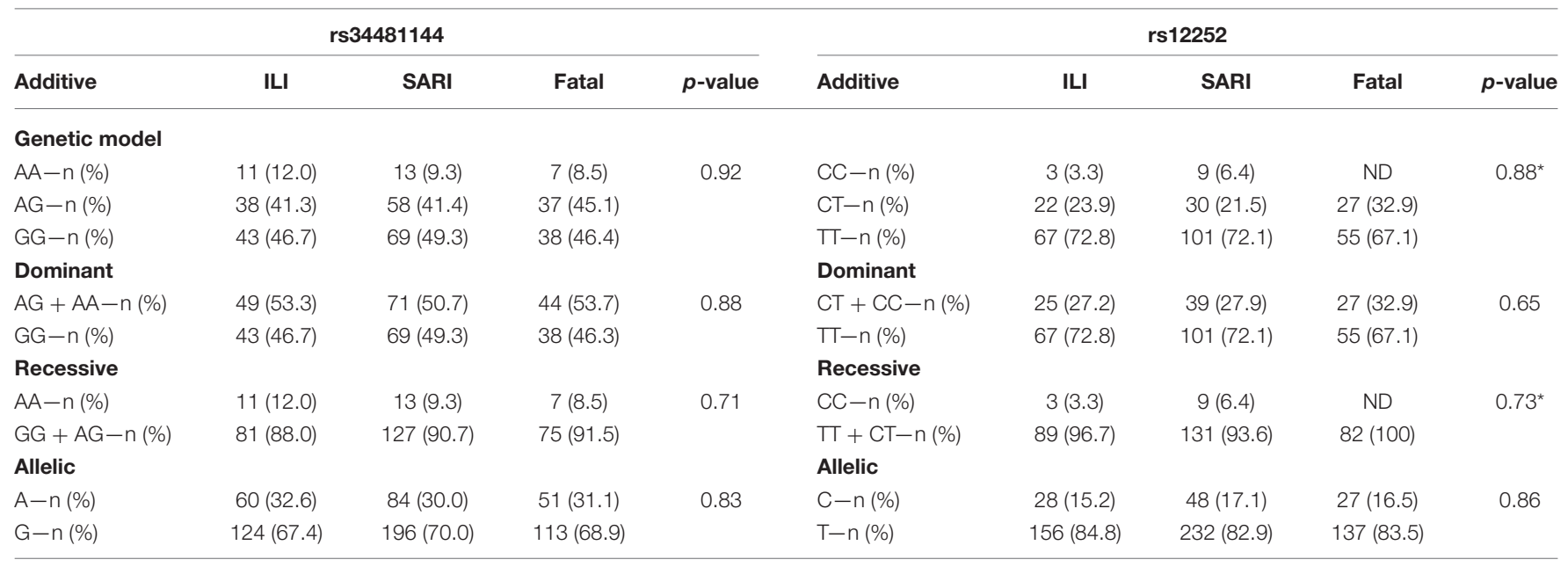

Statistical significance was considered when $p<0.05$ and was assessed by chi-square test. All comparisons were performed between the clinical groups (ILI, SARI, and Fatal), with exception to where symbol * appears (ILI vs. SARI + fatal). ILI, influenza-like illness; SARI, severe acute respiratory infection; ND, not detected.
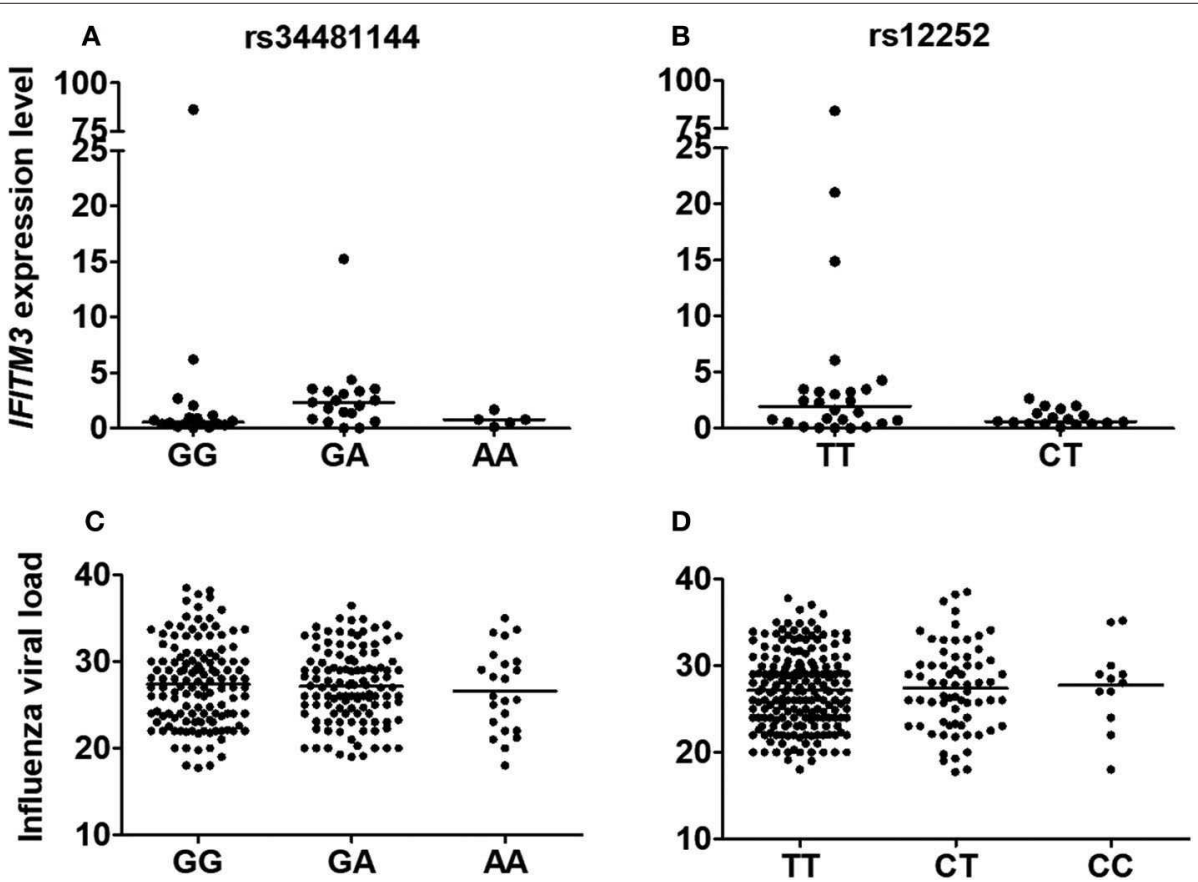

FIGURE 2 | Relationships between rs34481144 and rs12252 genotypes with IFITM3 expression levels [(A,B), respectively] and with influenza A(H1N1)pdm09 viral load [(C,D), respectively] in human respiratory clinical samples. IFITM3 expression level was assessed by quantitative real time PCR ( $\triangle \Delta C T$ method) by using GAPDH as a housekeeping gene. Influenza viral load was determined by real-time RT-PCR CT (cycle threshold) number during its detection. Bars represent median.

can restrict the replication of influenza viruses (Brass et al., 2009; Everitt et al., 2013), we evaluated the correlation between IFITM3 levels and influenza viral load, regardless of the genotype. However, the analysis showed a poor correlation coefficient $\left(R^{2}=0.02 ; p=0.34\right.$; Supplementary Figure $1 \mathrm{~A}$ and Supplementary Table 1). Moreover, IFITM3 expression in the respiratory specimens showed no differential levels in the different clinical groups ILI, SARI and fatal (Supplementary Figure 1B).

\section{DISCUSSION}

The antiviral restriction factor IFITM3 acts as part of innate immune response initial steps to limit influenza infection. It halts the formation of viral membrane fusion pores with host endosomes, by clustering in the IAV containing endosomes, impairing entry into the cytosol and subsequent viral replication (Kummer et al., 2019; Suddala et al., 2019). In addition, IFITM3 has also been shown to be induced, in DCs, after influenza 
infection, in mice, and is involved in migration of these cells to lymph nodes and consequent activation of influenza-specific lymphocytes (Infusini et al., 2015). Additionally, resident lung CD8+ T memory cells present persistent higher levels of IFITM3, which protect these cells to secondary influenza infections (Wakim et al., 2013). Because of these pivotal roles during immune response, some authors have suggested that genetic variation in the IFITM3 gene could affect its function. Some precedent studies have investigated rs $34481144 \mathrm{~A}$ and rs12252C distribution in the context of influenza infection and its clinical progression (Jia et al., 2012; Everitt et al., 2013; Allen et al., 2018).

Herein, we examined the frequency of such SNPs in Brazilian human clinical respiratory samples collected from influenza $\mathrm{A}(\mathrm{H} 1 \mathrm{~N} 1) \mathrm{pdm} 09$ positive cases during the post-pandemic period, from 2012 to 2018. Although the frequency of rs34481144A in our influenza cohort $(31,1 \%)$ was substantially higher than those previously described for healthy subjects from the Americas region (23.3\%; Auton et al., 2015), the presence of this polymorphism was not associated with severe clinical outcomes nor with IFITM3 expression levels and influenza viral load in respiratory samples. These findings suggest that SNP rs34481144A could even favor a higher susceptibility to viral infection, however, does not account for the development of severe viral infection. The results observed in our cohort are opposed to those reported by Allen et al., who found that rs34481144A was associated with severe influenza infection in three cohorts, as well as showed a reduction of IFITM3 levels in PBMCs from A allele carriers (Allen et al., 2018). It is noteworthy to point out that the epithelial cells of the respiratory tract are the initial target of influenza infection, and therefore constitute a good model for assessing the level of expression of IFITM3 during influenza infection. The different results found in our study and in the study by Allen et al. are probably due to the different cellular types used in the studies. Conversely, a different study described that $\mathrm{rs} 34481144 \mathrm{~A}$ had a protective effect against severe viral infection under the dominant model, as well as similar IFITM3 promoter activity when comparing rs34481144 WT and mutant HeLa cells (David et al., 2018). This last study demonstrated that rs34481144A occurrence in Portuguese influenza positive individuals $(31.1 \%)$ was highly similar to our Brazilian positive cohort (30.5\%), additionally to a similar frequency of allele A between severely affected Brazilian patients (30.4\%) and the Portuguese (22.7\%), a likely result due to our deep historical relationship, as a former Portuguese colony. It also corroborates our results on the similarity of IFITM3 expression, despite the genotype. Further functional studies in representative cohorts of different geographical areas, involving several cellular types and in vitro models are necessary to better elucidate the effective rs34481144A role in influenza infection.

Similarly, we found no association between the presence of rs12252C and clinical outcomes of influenza infection, IFITM3 expression levels and influenza viral load in our specimens. In our study, the rs12252 CC genotype was not detected in patients in the fatal group, probably due to the low frequency of this genotype in our cohort. Earlier studies had detected a positive correlation between $\mathrm{rs} 12252 \mathrm{C}$ and disease severity, mainly in Chinese cohorts (Zhang et al., 2015; Chan et al., 2017; Pan et al., 2017) whereas research conducted with others cohorts, such as Europeans, Americans, Afro-Americans and Korean failed to identify such association (Mills et al., 2014; Gaio et al., 2016; Kim and Jeong, 2017; Randolph et al., 2017). The overall allelic frequency of rs12252C in our Brazilian influenza $\mathrm{A}(\mathrm{H} 1 \mathrm{~N} 1)$ pdm09 cohort (16.4\%) was lower than those reported for Chinese infected cohorts (69.4 and 65.7\%; Zhang et al., 2013; Pan et al., 2017), but showed comparable frequency with a Portuguese cohort (9.8\%; David et al., 2018), also reinforcing genetic inheritance similarities between Brazil and Portugal. The same trend was observed when rs $12252 \mathrm{C}$ allelic frequency was compared in severely ill patients, as the Brazilian cohort (16.9\%) has a similar prevalence as the Portuguese (9.1\%), whereas is inferior to the Chinese ones (76.5 and $81.2 \%$ ). Under a complementary perspective, rs $12252 \mathrm{C}$ total allelic rate in an American healthy cohort (17.7\%) is lower than an Eastern Asian one (53.0\%; Auton et al., 2015). Therefore, a speculative explanation for this set of observations could be that allelic frequency of rs $12252 \mathrm{C}$ is considerably higher among the Chinese population, when compared to others.

A very provocative finding of this report was that the recessive genotypes of both SNPs were not detected together and were always inherited with the dominant genotype of the other locus. This outcome was also previously described by others (Randolph et al., 2017; Allen et al., 2018; David et al., 2018). In addition, we showed that the geographical distribution of the rs34481144 and rs12252 alleles genotypes had opposed trends, as Brazilian infected individuals from the South presented the highest rs34481144A allele frequency (34.6\%) but the lowest rs12252C one (14.1\%). On a similar fashion, the lowest frequency of rs34481144A allele in the Northeast was matched with the highest $\mathrm{rs} 12252 \mathrm{C}$ allele frequency. Moreover, the frequency of rs $34481144 \mathrm{~A}$ in healthy population from the Americas (23.0\%) was higher than those found in Africa (4.0\%) and Eastern Asia (1.0\%). In contrast, rs12252C frequency in the Americas (17.7\%) was lower than in Africa (26.\%) and East Asia (53.0\%) (Auton et al., 2015). Altogether, these observations regarding opposite inheritance also related to geographical trends suggest that these alleles are in a strong linkage disequilibrium (LD), as also pointed out by others (Randolph et al., 2017; Allen et al., 2018; David et al., 2018). The LD pattern of such loci could be affected by natural selection, genetic drift, recombination and mutation (Slatkin, 2008). However, once we did not observe a relationship between these SNPs and IFITM3 expression levels in our analysis, further investigations need to be performed to better elucidate the functional correlation of these two loci inheritance patterns.

Considering the lack of connection between the genotypes and IFITM3 expression, we further noted that IFITM3 transcript levels, in our respiratory specimens, were not correlated with influenza viral load nor with clinical status of the influenza cases. This observation would likewise suggest that IFITM3 levels, in the moment of diagnosis, in this type of samples, are not related to the disease outcome and could not be used as a molecular biomarker for the disease progression.

In conclusion, our results showed no association of rs34481144A and rs12252C with severity or mortality of influenza infection nor with IFITM3 transcript levels and influenza viral load in upper respiratory tract samples in the Brazilian infected cohort studied. Subsequent functional studies 
would add valuable information on the role of these SNPs during influenza infection.

\section{DATA AVAILABILITY STATEMENT}

The raw data supporting the conclusions of this article will be made available by the authors, without undue reservation, to any qualified researcher.

\section{ETHICS STATEMENT}

The studies involving human participants were reviewed and approved by Fiocruz-IOC Ethics Committee. Necessity of written informed consent was avoided in this study because the participants were evaluated under the Brazilian ISS, as approved by the local Ethics Comittee.

\section{AUTHOR CONTRIBUTIONS}

JM and AM designed the experiments. JM, CG, and AM conducted the experiments. JM, MO, and AM performed the statistical analysis. JM, MO, CG, MS, and AM contributed to the writing and editing of the manuscript. $\mathrm{AM}$ and $\mathrm{MS}$ contributed with funding acquisition. All authors contributed to the article and approved the submitted version.

\section{FUNDING}

This work was supported by grants from the PAPES VII program from the Conselho Nacional de Desenvolvimento Científico e Tecnológico (CNPq) and GABRIEL's Young Scientist Award by Fondation Merieux granted to AM. Coordenação de

\section{REFERENCES}

Allen, E. K., Randolph, A. G., Bhangale, T., Dogra, P., Oshansky, C. M., Zamora, A. E., et al. (2018). SNP-mediated disruption of CTCF binding at the IFITM3 promoter is associated with severe influenza risk in humans. Nat. Med. 23, 975-983. doi: 10.1038/nm.4370

Alves-Silva, J., da Silva Santos, M., Guimarães, P. E. M., Ferreira, A. C. S., Bandelt, H. J., Pena, S. D. J., et al. (2000). The ancestry of Brazilian mtDNA lineages. Am. J. Hum. Genet. 67, 444-461. doi: 10.1086/303004

Auton, A., Abecasis, G. R., Altshuler, D. M., Durbin, R. M., Bentley, D. R., Chakravarti, A., et al. (2015). A global reference for human genetic variation. Nature 526, 68-74. doi: 10.1038/nature15393

Behillil, S., May, F., Fourati, S., Luyt, S.-E., Chicheportiche, T, Sonneville, R., et al. (2018). Oseltamivir resistance in severe influenza A(H1N1)pdm09 pneumonia and acute respiratory distress syndrome: a French multicenter observational cohort study. J. Med. Virol. 5, 836-843. doi: 10.1093/cid/ciz904

Brass, A. L., Huang, I. C., Benita, Y., John, S. P., Krishnan, M. N., Feeley, E. M., et al. (2009). The IFITM proteins mediate cellular resistance to influenza A H1N1 virus, west nile virus, and dengue virus. Cell 139, 1243-1254. doi: 10.1016/j.cell.2009.12.017

Chan, P. K. S., Lee, N., Cao, B., Ke, C., Lu, H., Hu, Y., et al. (2017). IFITM3, TLR3, and CD55 Gene SNPs and cumulative genetic risks for severe outcomes in chinese patients with H7N9/H1N1 pdm09 influenza. J. Infect. Dis. 216, 97-104. doi: 10.1093/infdis/jix235
Aperfeiçoamento de Pessoal de Nível Superior (CAPES—finance code 001) supported JM. Further support was given by the Coordenação Geral de Laboratórios de Saúde Pública (CGLab) Brazilian $\mathrm{MoH}$. The funders had no role in study design, data collection and interpretation, or the decision to submit the work for publication.

\section{ACKNOWLEDGMENTS}

We would like to thank the Brazilian Influenza Surveillance System for collection of samples and diagnosis of influenza $\mathrm{A}(\mathrm{H} 1 \mathrm{~N} 1) \mathrm{pdm} 09$ in most of the clinical samples. Thanks are due to technical assistance of additional members of the Laboratório de Vírus Respiratórios e do Sarampo-IOCFiocruz for additional diagnosis of influenza A(H1N1)pdm09 in some clinical samples.

\section{SUPPLEMENTARY MATERIAL}

The Supplementary Material for this article can be found online at: https://www.frontiersin.org/articles/10.3389/fcimb. 2020.00352/full\#supplementary-material

Supplementary Figure 1 | IFITM3 expression level in human respiratory clinical samples. (A) Correlation analysis of IFITM3 expression level and influenza viral load was performed by linear regression. R2 and $p$-value were calculated. Expression was assessed by quantitative real time PCR ( $\Delta \Delta C T$ method). GAPDH was used as the housekeeping gene. (B) IFITM3 expression level in clinical samples classified according to influenza severity as ILI, SARI and fatal cases. Bars represent median.

Supplementary Table 1 | Data used for the correlation analysis of IFITM3 expression level and influenza viral load. Influenza viral load was assessed as a measure of RT-PCR cycle threshold (CT) for detection of influenza A H1Pdm09 hemagglutinin (HA) gene and IFITM3 relative expression level, for each sample.

Cheng, Z., Zhou, J., To, K. K. W., Chu, H., Li, C., Wang, D., et al. (2015). Identification of TMPRSS2 as a susceptibility gene for severe 2009 pandemic $\mathrm{A}(\mathrm{H} 1 \mathrm{~N} 1)$ influenza and A(H7N9) influenza. J. Infect. Dis. 212, 1214-1221. doi: $10.1093 /$ infdis/jiv246

Chesarino, N. M., Mcmichael, T. M., Hach, J. C., and Yount, J. S. (2014). Phosphorylation of the antiviral protein interferon-inducible transmembrane protein 3 (IFITM3) dually regulates its endocytosis and ubiquitination. J. Biol. Chem. 289, 11986-11992. doi: 10.1074/jbc.M114.557694

Chesarino, N. M., McMichael, T. M., and Yount, J. S. (2015). E3 ubiquitin ligase NEDD4 promotes influenza virus infection by decreasing levels of the antiviral protein IFITM3. PLoS Pathog. 1:e5095. doi: 10.1371/journal.ppat. 1005095

Chutinimitkul, S., Herfst, S., Steel, J., Lowen, A. C., Ye, J., van Riel, D., et al. (2010). Virulence-associated substitution D222G in the hemagglutinin of 2009 pandemic influenza $\mathrm{A}(\mathrm{H} 1 \mathrm{~N} 1)$ virus affects receptor binding. J. Virol. 84, 11802-11813. doi: 10.1128/JVI.01136-10

Compton, A. A., Roy, N., Porrot, F., Billet, A., Casartelli, N., Yount, J. S., et al. (2016). Natural mutations in IFITM3 modulate post-translational regulation and toggle antiviral specificity. EMBO Rep. 17, 1657-1671. doi: 10.15252/embr.201642771

Cox, N. J., and Subbarao, K. (1999). Influenza. Lancet 354, 1277-1282. doi: 10.1016/S0140-6736(99)01241-6

Daley, P., Castriciano, S., Chernesky, M., and Smieja, M. (2006). Comparison of flocked and rayon swabs for collection of respiratory epithelial cells 
from uninfected volunteers and symptomatic patients. J. Clin. Microbiol. 44, 2265-2267. doi: 10.1128/JCM.02055-05

Danielle Iuliano, A., Roguski, K. M., Chang, H. H., Muscatello, D. J., Palekar, R., Tempia, S., et al. (2018). Estimates of global seasonal influenza-associated respiratory mortality: a modelling study HHS public access. Lancet 391, 1285-1300. doi: 10.1016/S0140-6736(17)33293-2

David, S., Correia, V., Antunes, L., Faria, R., Ferrão, J., Faustino, P., et al. (2018). Population genetics of IFITM3 in Portugal and Central Africa reveals a potential modifier of influenza severity. Immunogenetics 70, 169-177. doi: $10.1007 / \mathrm{s} 00251-017-1026-2$

Desai, T. M., Marin, M., Chin, C. R., Savidis, G., Brass, A. L., and Melikyan, G. B. (2014). IFITM3 restricts influenza a virus entry by blocking the formation of fusion pores following virus-endosome hemifusion. PLoS Pathog. 10:e4048. doi: 10.1371/journal.ppat.1004048

Epperson, S., Davis, C. T., Brammer, L., Abd Elal, A. I., Ajayi, N., Barnes, J., et al. (2019). Update: influenza activity - United States and Worldwide, May 19-September 28, 2019, and composition of the 2020 Southern hemisphere influenza vaccine. MMWR. Morb. Mortal. Wkly. Rep. 68, 880-884. doi: $10.15585 / \mathrm{mmwr} . \mathrm{mm} 6840 \mathrm{a} 3$

Everitt, A. R., Clare, S., Pertel, T., John, S. P., Wash, R. S., Smith, E., et al. (2013). IFITM3 restricts the morbidity and mortality associated with influenza. Nature 484, 519-523. doi: 10.1038/nature10921

Falcon, A., Cuevas, M. T., Rodriguez-Frandsen, A., Reyes, N., Pozo, F., Moreno, S., et al. (2015). CCR5 deficiency predisposes to fatal outcome in influenza virus infection. J. Gen. Virol. 96, 2074-2078. doi: 10.1099/vir.0.000165

Feeley, E. M., Sims, J. S., John, S. P., Chin, C. R., Pertel, T., Chen, L. M., et al. (2011). IFITM3 inhibits influenza a virus infection by preventing cytosolic entry. PLoS Pathog. 7:e2337. doi: 10.1371/journal.ppat.1002337

Fislová, T., and Kostolanský, F. (2005). The factors of virulence of influenza a virus. Acta Virol. 49, 147-157. Available online at: http://www.elis.sk/download_file. php?product_id=117\&session_id=nv49okq42hbl3nkba9ois569q4

Gaio, V., Nunes, B., Pechirra, P., Conde, P., Guiomar, R., Dias, C. M., et al. (2016). Hospitalization risk due to respiratory illness associated with genetic variation at IFITM3 in patients with influenza A(H1N1)pdm09 infection: a case-control study. PLoS ONE 11:e158181. doi: 10.1371/journal.pone.0158181

Garcia, C. C., Tavares, L. P., Dias, A. C. F., Kehdy, F., Alvarado-arnez, L. E., Queiroz-junior, C. M., et al. (2018). Phosphatidyl inositol 3 Kinase- gamma balances antiviral and inflammatory responses during influenza a H1N1 infection : from murine model to genetic association in patients. Front. Immunol. 9:975. doi: 10.3389/fimmu.2018.00975

Heil, F., Hemmi, H., Hochrein, H., Ampenberger, F., Kirschning, C., Akira, S., et al. (2004). Species-specific recognition of single-stranded RNA via till-like receptor 7 and 8. Science 80, 1526-1529. doi: 10.1126/science.1093620

Huang, I.-C., Bailey, C. C., Weyer, J. L., Radoshitzky, S. R., and Becker, M. M. (2011). Distinct patterns of IFITM-mediated restriction of filoviruses, SARS coronavirus, and influenza a virus. PLoS Pathog 7:1001258. doi: 10.1371/journal.ppat.1001258

Infusini, G., Smith, J. M., Yuan, H., Pizzolla, A., Ng, W. C., Londrigan, S. L., et al. (2015). Respiratory DC use IFITM3 to avoid direct viral infection and safeguard virus-specific CD8+ T cell priming. PLoS ONE 10:e143539. doi: 10.1371/journal.pone.0143539

Jia, R., Pan, Q., Ding, S., Rong, L., Liu, S.-L., Geng, Y., et al. (2012). The N-terminal region of IFITM3 modulates its antiviral activity by regulating IFITM3 cellular localization. J. Virol. 86, 13697-13707. doi: 10.1128/JVI.01828-12

Jia, R., Xu, F., Qian, J., Yao, Y., Miao, C., Zheng, Y.-M., et al. (2014). Identification of an endocytic signal essential for the antiviral action of IFITM3 NIH public access. Cell Microbiol. 16, 1080-1093. doi: 10.1111/cmi.12262

Kawai, T., and Akira, S. (2010). The role of pattern-recognition receptors in innate immunity: update on toll-like receptors. Nat. Immunol. 11, 373-384. doi: $10.1038 /$ ni. 1863

Kim, N., Now, H., Nguyen, N. T. H., and Yoo, J. Y. (2016). Multilayered regulations of RIG-I in the anti-viral signaling pathway. J. Microbiol. 54, 583-587. doi: 10.1007/s12275-016-6322-2

Kim, Y.-C., Jeong, M.-J., and Jeong, B.-H. (2019). Strong association of regulatory single nucleotide polymorphisms (SNPs) of the IFITM3 gene with influenza H1N1 2009 pandemic virus infection. Cell. Mol. Immunol. 3-5. doi: 10.1038/s41423-019-0322-1

Kim, Y. C., and Jeong, B. H. (2017). No correlation of the disease severity of influenza a virus infection with the rs 12252 polymorphism of the interferon-induced transmembrane protein 3 gene. Intervirology 60, 69-74. doi: $10.1159 / 000479087$

Kummer, S., Avinoam, O., and Kräusslich, H. G. (2019). IFITM3 clusters on virus containing endosomes and lysosomes early in the influenza a infection of human airway epithelial cells. Viruses 11:548. doi: 10.3390/v11060548

Liu, Y., Childs, R. A., Matrosovich, T., Wharton, S., Palma, A. S., Chai, W., et al. (2010). Altered receptor specificity and cell tropism of D222G hemagglutinin mutants isolated from fatal cases of pandemic $\mathrm{A}(\mathrm{H} 1 \mathrm{~N} 1) 2009$ influenza virusं ‡. J. Virol. 84, 12069-12074. doi: 10.1128/JVI.01639-10

Makvandi-Nejad, S., Laurenson-Schafer, H., Wang, L. L., Wellington, D., Zhao, Y., Jin, B., et al. (2018). Lack of truncated IFITM3 transcripts in cells homozygous for the rs12252-C variant that is associated with severe influenza infection. $J$. Infect. Dis. 217, 257-262. doi: 10.1093/infdis/jix512

Mares-Guia, M. A. M., de, M., Horta, M. A., Romano, A., Rodrigues, C. D. S., Mendonça, M. C. L., et al. (2020). Yellow fever epizootics in non-human primates, Southeast and Northeast Brazil (2017 and 2018). Parasit. Vectors 13:90. doi: 10.1186/s13071-020-3966-x

Matos, A. R., Martins, J. S. C. C., Oliveira, M., de, L. A., Garcia, C. C., and Siqueira, M. M. (2019). Human CCR5 $\Delta 32$ (rs333) polymorphism has no influence on severity and mortality of influenza $\mathrm{A}(\mathrm{H} 1 \mathrm{N1}) \mathrm{pdm} 09$ infection in Brazilian patients from the post pandemic period. Infect. Genet. Evol. 67, 55-59. doi: 10.1016/j.meegid.2018.10.024

Matos, A. R., Resende, P. C., Miranda, M. D., Garcia, C. C., Caetano, B. C., Lopes, J. C. O., et al. (2018). Susceptibility of Brazilian influenza A(H1N1)pdm09 viruses to neuraminidase inhibitors in the 2014-2016 seasons: identification of strains bearing mutations associated with reduced inhibition profile. Antiviral Res. 154, 35-43. doi: 10.1016/j.antiviral.2018.03.010

Mills, T. C., Rautanen, A., Elliott, K. S., Parks, T., Naranbhai, V., Ieven, M. M., et al. (2014). IFITM3 and susceptibility to respiratory viral infections in the community. J. Infect. Dis. 209, 1028-1031. doi: 10.1093/infdis/ jit468

Mychaleckyj, J. C., Havt, A., Nayak, U., Pinkerton, R., Farber, E., Concannon, P., et al. (2017). Genome-wide analysis in brazilians reveals highly differentiated native American genome regions. Mol. Biol. Evol. 34, 559-574. doi: 10.1093/molbev/msw249

Pan, Y., Yang, P., Dong, T., Zhang, Y., Shi, W., Peng, X., et al. (2017). IFITM3 Rs12252-C Variant increases potential risk for severe influenza virus infection in chinese population. Front. Cell. Infect. Microbiol. 7:294. doi: $10.3389 /$ fcimb.2017.00294

Randolph, A. G., Yip, W. K., Allen, E. K., Rosenberger, C. M., Agan, A. A., Ash, S. A., et al. (2017). Evaluation of IFITM3 rs12252 association with severe pediatric influenza infection. J. Infect. Dis. 216, 14-21. doi: 10.1093/infdis/ jix 242

Rodriguez, S., Gaunt, T. R., and Day, I. N. M. (2009). Hardy-Weinberg equilibrium testing of biological ascertainment for Mendelian randomization studies. Am. J. Epidemiol. 169, 505-514. doi: 10.1093/aje/kwn359

Secretaria de Vigilância em Saúde (2012). Informe Técnico De Influenza. Edição 1.

Slatkin, M. (2008). Linkage disequilibrium - Understanding the evolutionary past and mapping the medical future. Nat. Rev. Genet. 9, 477-485. doi: $10.1038 /$ nrg2 2361

Suddala, K. C., Lee, C. C., Meraner, P., Marin, M., Markosyan, R. M., Desai, T. M., et al. (2019). Interferon-induced transmembrane protein 3 blocks fusion of sensitive but not resistant viruses by partitioning into virus-carrying endosomes. PLoS Pathog. 15:e1007532. doi: 10.1371/journal.ppat.1007532

Takashita, E., Daniels, R. S., Fujisaki, S., Gregory, V., Gubareva, L. V., Huang, W., et al. (2020). Global update on the susceptibilities of human influenza viruses to neuraminidase inhibitors and the cap-dependent endonuclease inhibitor baloxavir, 2017-2018. Antiviral Res. 175, 104718. doi: 10.1016/j.antiviral.2020.104718

Tarnow, C., Engels, G., Arendt, A., Schwalm, F., Sediri, H., Preuss, A., et al. (2014). TMPRSS2 is a host factor that is essential for pneumotropism and pathogenicity of H7N9 influenza a virus in mice. J. Virol. 88, 4744-4751. doi: 10.1128/JVI.03799-13

Taubenberger, J. K., and Morens, D. M. (2008). The Pathology of Influenza Virus Infections. Annu. Rev. Pathol. 3, 499-522. doi: 10.1146/annurev.pathmechdis.3.121806.154316

van der Sandt, C. E., Kreijtz, J. H. C. M., and Rimmelzwaan, G. F. (2012). Evasion of influenza A viruses from innate and adaptive immune responses. Viruses 4, 1438-1476. doi: 10.3390/v4091438 
Wakim, L. M., Gupta, N., Mintern, J. D., and Villadangos, J. A. (2013). Enhanced survival of lung tissue-resident memory CD8 $+\mathrm{T}$ cells during infection with influenza virus due to selective expression of IFITM3. Nat. Immunol. 14, 238-245. doi: $10.1038 /$ ni.2525

WHO (2020). Global Influenza Surveillance and Response System (GISRS). WHO World Health Organization (2019). Global Influenza Strategy 2019-2030.

Zani, A., and Yount, J. S. (2018). Antiviral protection by IFITM3 in vivo HHS public access. Curr. Clin. Microbiol. Rep. 5, 229-237. doi: 10.1007/s40588-018-0103-0

Zhang, Y., Makvandi-Nejad, S., Qin, L., Zhao, Y., Zhang, T., Wang, L., et al. (2015). Interferon-induced transmembrane protein-3 rs12252$\mathrm{C}$ is associated with rapid progression of acute HIV-1 infection in Chinese MSM cohort. AIDS 29, 889-894. doi: 10.1097/QAD.00000000000 00632

Zhang, Y.-H., Zhao, Y., Li, N., Peng, Y.-C., Giannoulatou, E., Jin, R.-H., et al. (2013). Interferon-induced transmembrane protein-3 genetic variant rs12252-C is associated with severe influenza in Chinese individuals. Nat.
Commun. 4:1418. doi: 10.1038/ncomms2433

Zhou, J., To, K. K. W., Dong, H., Cheng, Z. S., Lau, C. C. Y., Poon, V. K. M., et al. (2012). A functional variation in CD55 increases the severity of 2009 pandemic H1N1 influenza a virus infection. J. Infect. Dis. 206, 495-503. doi: 10.1093/infdis/jis378

Conflict of Interest: The authors declare that the research was conducted in the absence of any commercial or financial relationships that could be construed as a potential conflict of interest.

Copyright (อ 2020 Martins, Oliveira, Garcia, Siqueira and Matos. This is an openaccess article distributed under the terms of the Creative Commons Attribution License (CC BY). The use, distribution or reproduction in other forums is permitted, provided the original author(s) and the copyright owner(s) are credited and that the original publication in this journal is cited, in accordance with accepted academic practice. No use, distribution or reproduction is permitted which does not comply with these terms. 